\title{
Network Pharmacology Analysis and Molecular Characterization of the Herbal Medicine Formulation Qi-Fu-Yin for the Inhibition of the Neuroinflammatory Biomarker iNOS in Microglial BV-2 Cells: Implication for the Treatment of Alzheimer's Disease
}

\author{
Fung Yin Ngo, ${ }^{1}$ Weiwei Wang, ${ }^{1,2}$ Qilei Chen, ${ }^{3}$ Jia Zhao, ${ }^{1}$ Hubiao Chen, ${ }^{3}$ Jin-Ming Gao $\mathbb{D},{ }^{2}$ \\ and Jianhui Rong $\mathbb{D}^{1,4}$ \\ ${ }^{1}$ School of Chinese Medicine, The University of Hong Kong, Hong Kong 999077, China \\ ${ }^{2}$ Shaanxi Key Laboratory of Natural Products \& Chemical Biology, College of Chemistry \& Pharmacy, Northwest A\&F University, \\ Yangling 712100, China \\ ${ }^{3}$ School of Chinese Medicine, Hong Kong Baptist University, Hong Kong 999077, China \\ ${ }^{4}$ Shenzhen Institute of Research and Innovation, The University of Hong Kong, Shenzhen 518057, China
}

Correspondence should be addressed to Jin-Ming Gao; jinminggao@nwsuaf.edu.cn and Jianhui Rong; jrong@hkucc.hku.hk

Received 10 June 2020; Accepted 12 August 2020; Published 1 September 2020

Academic Editor: Shi Yuan Xu

Copyright (C) 2020 Fung Yin Ngo et al. This is an open access article distributed under the Creative Commons Attribution License, which permits unrestricted use, distribution, and reproduction in any medium, provided the original work is properly cited.

\begin{abstract}
Aberrant microglial activation drives neuroinflammation and neurodegeneration in Alzheimer's disease (AD). The present study is aimed at investigating whether the herbal formula Qi-Fu-Yin (QFY) could inhibit the inflammatory activation of cultured BV-2 microglia. A network pharmacology approach was employed to predict the active compounds of QFY, protein targets, and affected pathways. The representative pathways and molecular functions of the targets were analyzed by Gene Ontology (GO) and pathway enrichment. A total of 145 active compounds were selected from seven herbal ingredients of QFY. Targets (e.g., MAPT, APP, ACHE, iNOS, and COX-2) were predicted for the selected active compounds based on the relevance to AD and inflammation. As a validation, fractions were sequentially prepared by aqueous extraction, ethanolic precipitation, and HPLC separation, and assayed for downregulating two key proinflammatory biomarkers iNOS and COX-2 in lipopolysaccharide(LPS-) challenged BV-2 cells by the Western blotting technique. Moreover, the compounds of QFY in $90 \%$ ethanol downregulated iNOS in BV-2 cells but showed no activity against COX-2 induction. Among the herbal ingredients of QFY, Angelicae Sinensis Radix and Ginseng Radix et Rhizoma contributed to the selective inhibition of iNOS induction. Furthermore, chemical analysis identified ginsenosides, especially Rg3, as antineuroinflammatory compounds. The herbal formula QFY may ameliorate neuroinflammation via downregulating iNOS in microglia.
\end{abstract}

\section{Introduction}

Alzheimer's disease (AD) is the major neurodegenerative cause of progressive dementia in the elderly [1]. The pathology of $\mathrm{AD}$ is hallmarked by the accumulation of extracellular $\beta$-amyloid $(\mathrm{A} \beta)$ and the formation of peptide plaques and intraneuronal tau lesions, resulting in impaired neurotransmission and neuronal death [2]. Among the existing pharmaco- logical anti-AD interventions, acetylcholinesterase inhibitors are known to restore cholinergic neurotransmission, whereas $N$-methyl-D-aspartate (NMDA) receptor antagonists suppress the neuronal excitability towards NMDA [3]. However, some controversial results showed that these agents barely prevented the progression of $\mathrm{AD}$ and could cause adverse effects [4]. Nevertheless, neurotoxic peptide $\mathrm{A} \beta$ activates microglia and exacerbates neuroinflammation, leading to 
the onset and progression of $\mathrm{AD}[5,6]$. Indeed, the overexpression of proinflammatory enzymes such as inducible nitric oxide synthase (iNOS) and cyclooxygenase-2 (COX2) jeopardizes the survival of neurons in brains [7]. Notably, excessive nitric oxide in the brain induced oxidative damage in neurons and led to the activation of apoptosis [8]. Therefore, effective inhibition of neuroinflammation represents a key strategy for the management of $\mathrm{AD}$, but the in vivo efficacies of antineuroinflammatory and microglia-targeting agents remain uncertain [9].

Traditional herbal medicines may serve as alternative therapeutic strategies against various multifactorial and complex chronic diseases including AD [10]. The herbal formula Qi-Fu-Yin (QFY) was documented for managing dementia four hundred years ago [11]. The formula QFY is composed of seven herbal ingredients, namely, Atractylodis Macrocephalae Rhizoma (Baizhu, BZ), Angelicae Sinensis Radix (Danggui, DG), Glycyrrhizae Radix et Rhizoma (Gancao, GC), Ginseng Radix et Rhizoma (Renshen, RS), Rehmanniae Radix Preparata (Shudi, SD), Ziziphi Spinosae Semen (Suanzaoren, SZR), and Polygalae Radix (Yuanzhi, YZ). Previous studies showed that QFY improved learning and memory of $\mathrm{AD}$ rodents via increasing somatostatin in the hippocampus, reducing $\mathrm{A} \beta$ accumulation and proinflammatory biomarkers in mouse brains [12-14]. Comprehensive chemical profiling of QFY and a modified QFY formula with two additional herbs identified active compounds that were neuroprotective or exhibited a therapeutic effect towards AD [15, 16]. Several other studies also suggested the antineuroinflammatory action of GC, RS, and YZ via modulating microglial activity [17-19]. However, effort is needed to elucidate the molecular mechanisms by which QFY modulates microglial activation within the context of AD.

The aim of the present study was to characterize the antineuroinflammatory activity of QFY. We employed a network pharmacology approach to analyze the compound-target interactions of QFY and the relevant signaling pathways. We further validated the antineuroinflammatory property in microglial BV-2 cell culture and identified the principal active compounds through a bioactivity-guided fractionation procedure.

\section{Materials and Methods}

2.1. Chemicals and Reagents. The dried aqueous extracts of seven QFY ingredients were manufactured by Nong's Pharmaceutical Ltd., Hong Kong. Dulbecco's modified Eagle's medium (DMEM), fetal bovine serum (FBS), and penicillin/streptomycin solution were purchased from Invitrogen (Carlsbad, CA, USA). Protein assay dye reagent concentrate was purchased from Bio-Rad (Hercules, CA, USA). Lipopolysaccharide (LPS), RIPA assay buffer, protease inhibitor cocktail, and anti-rabbit horseradish peroxidase- (HRP-) conjugated IgG secondary antibody were purchased from Sigma-Aldrich (St. Louis, MO, USA). An antibody against iNOS was purchased from Abcam (Cambridge, UK). Antibodies against COX-2 and GAPDH were purchased from Cell Signaling Technology (Boston, MA, USA). Anti-mouse HRP-conjugated IgG was purchased from Santa Cruz Bio- technology (Santa Cruz, CA, USA). An enhanced chemiluminescence (ECL) detection reagent was purchased from GE Healthcare (Uppsala, Sweden). Ginsenoside Rg3 with the purity of $>98 \%$ was purchased from Nanjing Spring and Autumn Biological Engineering Company (Nanjing, China).

2.2. Identification of the Active Compounds in the Formulation QFY. The chemical compounds were selected from the herbs of QFY in the databases of TCMSP (https:// tcmspw.com/) based on the oral bioavailability $(O B) \geq 30 \%$, drug-likeness $(D L) \geq 0.18$, and blood-brain barrier $(B B B) \geq$ -0.3 . On the other hand, the compounds in the herbal ingredient not found in TCMSP were selected from the database of TCMID (http://www.megabionet.org/tcmid/) when the compounds possessed the blood-brain barrier (BBB) permeability of $\geq-1$ through checking pkCSM (http://biosig .unimelb.edu.au/pkcsm/) and did not violate more than one of the criteria stated in Lipinski's rule of five. In addition, the active compounds were collected by text mining.

2.3. Prediction of Protein Targets. The active compounds in the formulation QFY were loaded to the Similarity Ensemble Approach (SEA) at http://sea.bkslab.org, the Search Tool for Interactions of Chemicals (STITCH) at http://stitch.embl.de, and SwissTargetPrediction at http://swisstargetprediction.ch for the prediction of the corresponding biological targets. The AD-related targets after checking Molecule Annotation System 3 (MAS 3.0) at http://bioinfo.capitalbio.com/mas3, Therapeutic Targets Database (TTD) at http://bidd.nus.edu .sg/group/cjttd/, and Comparative Toxicogenomics Database (CTD) at http://ctdbase.org/ were retained for further study.

2.4. Gene Ontology and Pathway Enrichment Analysis. The selected targets were analyzed for the enriched Gene Ontology (GO) terms and the Kyoto Encyclopedia of Genes and Genomes (KEGG) pathway using the online bioinformatics tool DAVID 6.8 at http://david.ncifcrf.gov. The annotations with adjusted $p<0.05$ were considered significantly enriched.

2.5. Visualization of the Compound-Target Interaction and the Target-Pathway Relationship. The interaction networks were visualized using the open software package Cytoscape (http://www.cytoscape.org/). For the compound-target interactions, the active compounds in QFY and the corresponding protein targets were presented in a compound-target (C-T) network. For the target-pathway interactions, the targets and the related pathways were presented in a targetpathway (T-P) network.

2.6. Microglial BV-2 Cell Culture and Treatment. The murine microglial cell line BV-2 was purchased from the American Type Culture Collection (Manassas, VA, USA) and cultured in DMEM supplemented with $10 \%$ heat-inactivated FBS and $1 \%$ penicillin/streptomycin solution. The cells were incubated at an atmosphere with $5 \% \mathrm{CO}_{2}$ at $37^{\circ} \mathrm{C}$. For the indicated treatments, BV-2 cells were plated in 6-well plates at a density of $2 \times 10^{5} / \mathrm{mL}$ overnight. BV-2 cells were treated with herbal extracts as indicated and costimulated with $0.1 \mu \mathrm{g} / \mathrm{mL}$ LPS in DMEM supplemented with 3\% FBS for 24 hours. 
2.7. Fractionation of Herbal Extracts. QFY was prepared by mixing the dried aqueous extracts of seven herbal ingredients: BZ, 1.6 g; DG, $3 \mathrm{~g}$; GC, $0.6 \mathrm{~g}$; RS, $1.2 \mathrm{~g}$; SD, $1.8 \mathrm{~g}$; SZR, $1.2 \mathrm{~g}$; and $\mathrm{YZ}, 1 \mathrm{~g}$. Following extraction with distilled water at $80^{\circ} \mathrm{C}$ for $30 \mathrm{~min}$, the mixture was cooled to room temperature and centrifuged at $13,000 \mathrm{rpm}$ for $10 \mathrm{~min}$. The supernatant was recovered and precipitated with ethanol at the final concentrations of $50 \%, 75 \%$, and $90 \%$ overnight at $4^{\circ} \mathrm{C}$. After centrifugation at $6,000 \mathrm{rpm}$ for $30 \mathrm{~min}$, the supernatant was collected to yield the corresponding ethanol solutions of QFY. The ethanolic materials were dried with a rotary evaporator, dissolved in DMSO, and sterilized by passing through a $0.22 \mu \mathrm{m}$ syringe filter for bioassays. For HPLC separation, the compounds were separated on an ACE C18 HPLC column $(250 \times 4.6 \mathrm{~mm}, 5 \mu \mathrm{m})$ from Advanced Chromatography Technologies Ltd. (Aberdeen, Scotland, UK) under the control of the Waters Controller 600S HPLC system coupled with a photodiode array detector (Waters, Milford, MA, USA). The column temperature was maintained at $25^{\circ} \mathrm{C}$. The mobile phases of (A) methanol and (B) $0.1 \%$ aqueous formic acid were pumped into the column at a flow rate of $1.0 \mathrm{~mL} / \mathrm{min}$ to form the gradient as follows: $0-25 \mathrm{~min}, 5-$ $50 \% \mathrm{~A} ; 25-45 \mathrm{~min}, 50-70 \% \mathrm{~A} ; 45-47 \mathrm{~min}, 70-95 \% \mathrm{~A}$; and 47-53 $\mathrm{min}, 95 \% \mathrm{~A}$. The fractions were collected, dried, and dissolved in DMSO for subsequent bioassays.

2.8. Western Blot Analysis. After drug treatment as indicated, BV-2 cells were washed twice with cold PBS and lysed with cold RIPA buffer to collect the cellular proteins. The cellular proteins $(30 \mu \mathrm{g})$ from each sample were resolved by $12 \%$ SDS-polyacrylamide gel electrophoresis and transferred to a polyvinylidene difluoride (PVDF) membrane. After overnight blocking with 5\% non-fat milk, the membranes were probed with primary antibodies against iNOS, COX-2, and GAPDH overnight. The bound antibodies were detected with the HRP conjugate of the corresponding anti-rabbit or antimouse secondary antibodies for 2 hours. The activity of HRP was detected with ECL detection reagents. The blots were quantified by a densitometric method using the NIH ImageJ software.

2.9. Chemical Profiling by the LC-MS/MS System. The Agilent 6540 Ultra High Definition (UHD) Accurate-Mass Q-TOF LC/MS system from Agilent Technologies (Santa Clara, CA, USA) was used for mass spectrometry analysis. The compounds in $10 \mu \mathrm{L}$ were injected and separated on an ACE C18 HPLC column at a flow rate of $1.0 \mathrm{~mL} / \mathrm{min}$ and column temperature of $40^{\circ} \mathrm{C}$, by using the same gradient from the mobile phases of (A) $0.1 \%$ formic acid in water and (B) methanol as described for HPLC. The MS parameters were set as follows: electrospray ionization source (ESI) in a negative mode, nitrogen $\left(\mathrm{N}_{2}\right)$ as drying gas, flow rate at $8 \mathrm{~L} / \mathrm{min}$, gas temperature at $300^{\circ} \mathrm{C}$, nebulizer at $40 \mathrm{psi}$, sheath gas temperature at $350^{\circ} \mathrm{C}$, flow rate of sheath gas at $8 \mathrm{~L} / \mathrm{min}$, capillary voltage at $4.0 \mathrm{kV}$, end plate offset at $-500 \mathrm{~V}$, fragmentor at $150 \mathrm{~V}$, skimmer at $65 \mathrm{~V}$, Oct RF Vpp at $600 \mathrm{~V}$, scan range of $100-1700 \mathrm{~m} / \mathrm{z}$, and collision energy at $25 \mathrm{~V}$ for MS and $45 \mathrm{~V}$ for MS/MS, respectively. The data was analyzed on Agilent MassHunter Qualitative Analysis B.06.00 and Agilent
MassHunter Quantitative Analysis B.06.00 from Agilent Technologies (Santa Clara, CA, USA).

2.10. Statistical Analysis. Data was presented as mean $\pm S D$ of three replicates. The difference between two groups was analyzed by one-way ANOVA and Tukey's multiple comparison using the GraphPad Prism 7 software (La Jolla, CA, USA). A $p$ value of $<0.05$ was considered statistically significant.

\section{Results}

3.1. QFY Targets Key Proteins in Inflammation and $A D$ Pathogenesis. To characterize the biological targets of QFY in a comprehensive manner, we employed a network pharmacology approach to dissect the complex multicompound and multitarget interaction. A total of 812 compounds in QFY were firstly retrieved from TCMSP and TCMID. Based on the pharmacokinetic or physiochemical properties, 126 active compounds were obtained while another 19 compounds were added by searching the PubMed database for the implications in the treatment of $\mathrm{AD}$. The nomenclature of the active compounds was guided by PubChem, and the detailed information is listed in Supplementary Tables 1 and 2. The active compounds were further classified by the Medical Subject Headings (MeSH) classification system and text mining. The candidate compounds were chemically categorized into flavonoids, terpenoids, alkaloids, xanthones, phenylpropanoids, lipids, glycosides, carboxylic acids, phenolic compound, and amino acids. The protein targets were fished out using various predictive models (e.g., SEA, STITCH, and SwissTargetPrediction). As shown in Figure 1(a), 25 protein targets formed a total of 213 interactions with 96 active compounds. Specifically, MAPT as an important pathological target in $\mathrm{AD}$ showed the highest connectivity with 56 of the active compounds. Several active compounds in QFY showed interactions with other AD-relevant mediators (e.g., BACE1, APP, and SNCA), cholinergic neurotransmission (e.g., ACHE, CHRM1, CHRNA4, and CHRNB2), and inflammation (e.g., COX-2, eNOS, and iNOS). For the target-pathway network as shown in Figure 1(b), the protein targets were mapped to 14 different biological pathways or diseases in KEGG. Six protein targets (i.e., MAPT, BACE1, APP, SNCA, MAOA, and MAOB) were categorized in "Alzheimer's disease," and five targets (i.e., ACHE, CHRM1, CHRNA4, CHRNB2, and FOS) were grouped to "cholinergic synapse," while another three targets (i.e., CASP8, FOS, and COX-2) were grouped to "TNF signaling pathway".

To characterize the functional annotations of the protein targets, Gene Ontology (GO) and KEGG pathway enrichment analysis were carried out on the online bioinformatics tool DAVID. As shown in Figure 2, the inflammation- or AD-related GO terms appeared in the ten most significantly enriched GO terms from "biological process" and "molecular function," in particular, acetylcholinesterase activity (GO: 0003990), amyloid-beta binding (GO: 0001540), and response to lipopolysaccharide (GO: 0032496). The three most significantly enriched KEGG pathways were Alzheimer's disease (KEGG: hsa05010), cholinergic neurotransmission pathway 


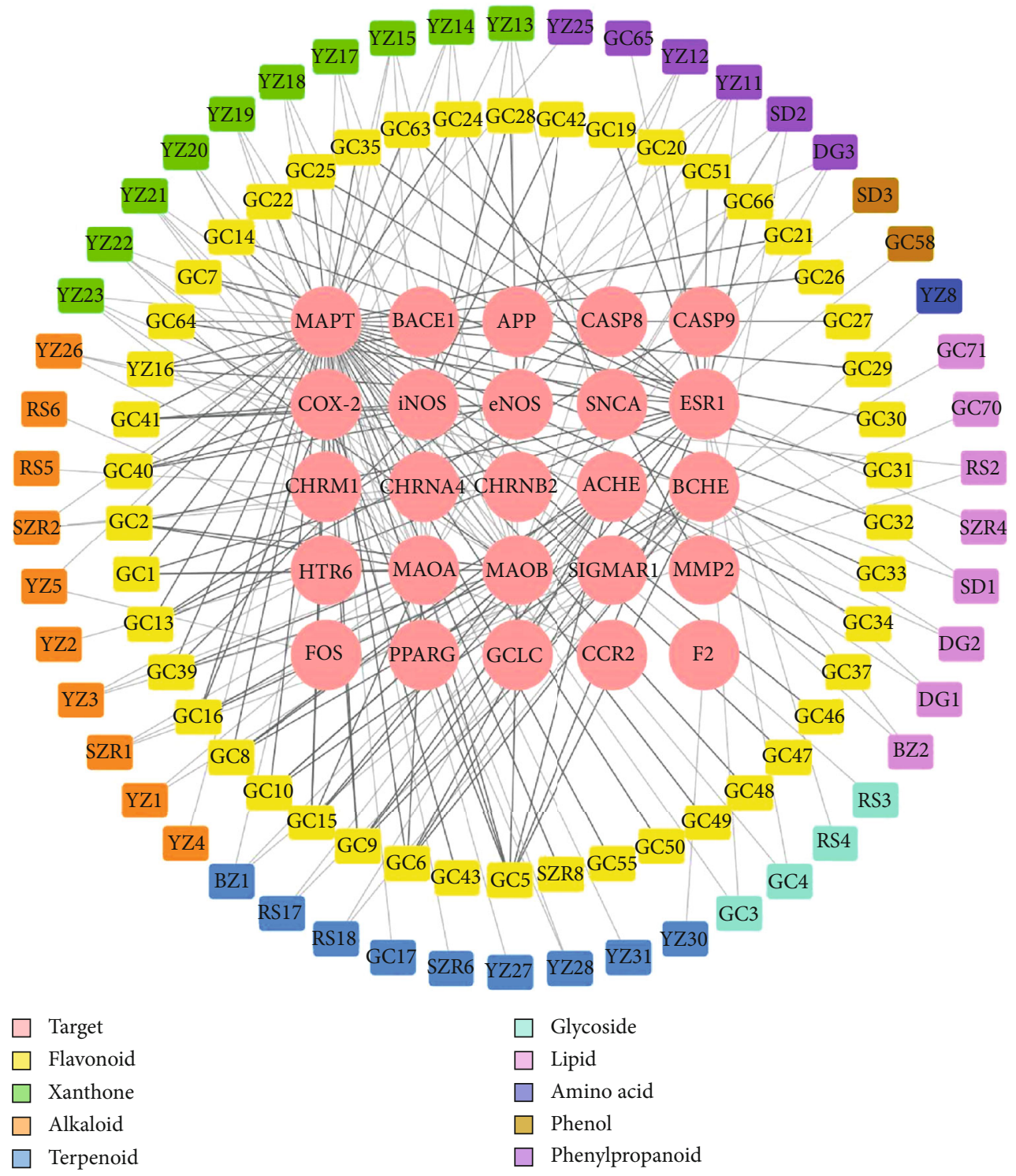

(a)

Figure 1: Continued. 


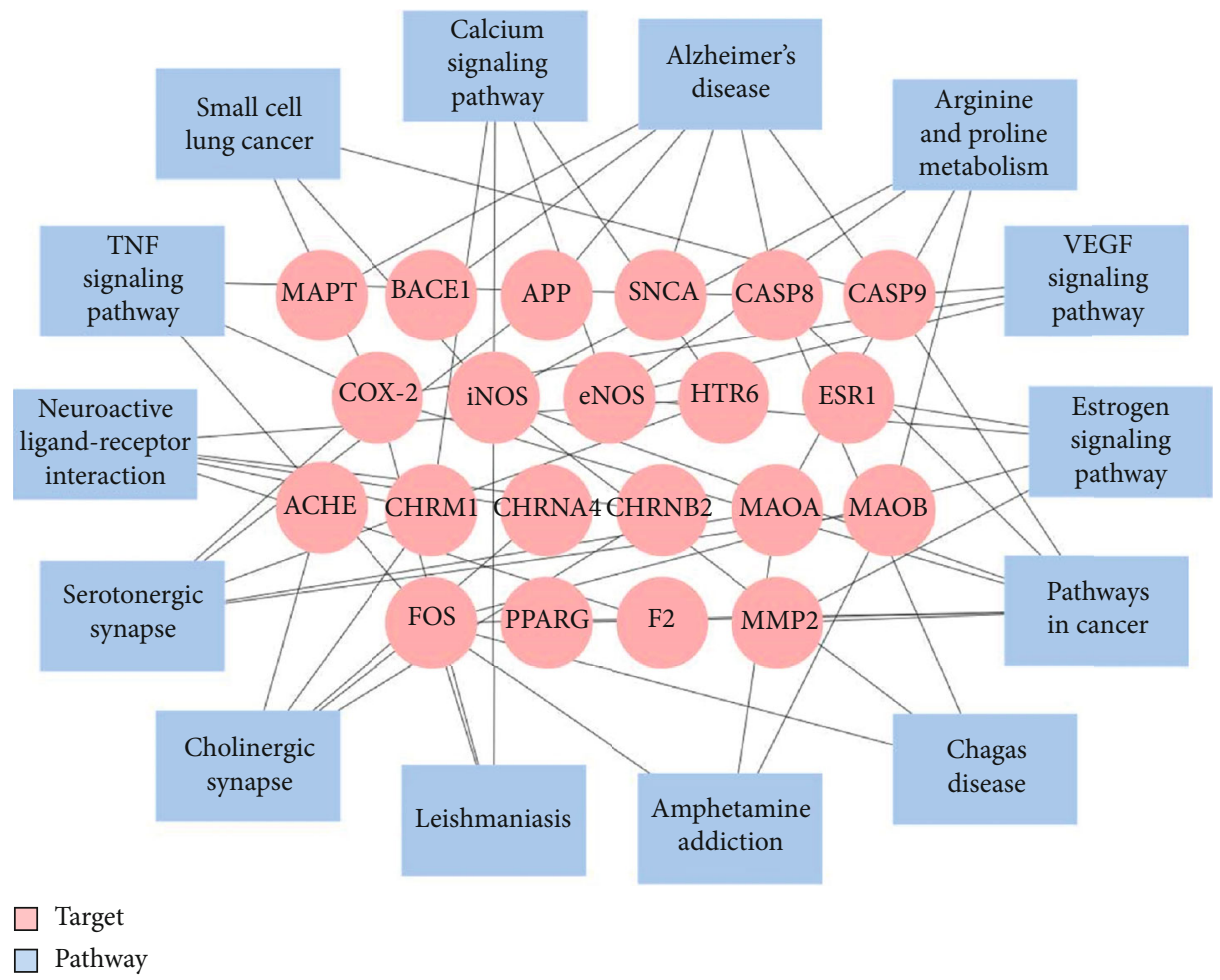

(b)

FIGURE 1: Network pharmacology analysis of QFY. (a) The compound-target (C-T) network. The grey lines indicate the interactions between the candidate active compounds (outer circles) from the individual herbal ingredients of QFY and the predicted protein targets at the center. (b) The target-pathway (T-P) network. The grey lines indicate the interactions between the protein targets at the center and the signaling pathways or diseases (outer circles).

(KEGG: hsa04725), and serotonergic neurotransmission pathway (KEGG: hsa04726).

\subsection{QFY Differentially Affected iNOS and COX-2 Expression} against LPS Stimulation. To validate the antineuroinflammatory action of QFY and identify the corresponding active compounds, we developed a bioactivity-guided fractionation approach involving ethanolic precipitation, HPLC separation, and in vitro assays (Figure 3(a)). The aqueous extract of QFY was firstly precipitated in $50 \%, 75 \%$, and $90 \%$ ethanol. The ethanol solutions and aqueous extract of QFY were assayed for the effects on iNOS and COX-2 in LPSstimulated BV-2 cells. As shown in Figure 3(b), LPSinduced iNOS expression was decreased by these ethanol solutions of QFY. The $90 \%$ ethanol solution of QFY (90\% QFY) selectively suppressed LPS-induced iNOS expression in a concentration-dependent manner. In contrast, none of the four QFY preparations effectively suppressed LPSinduced COX-2 expression. To identify the specific compounds, 90\% QFY preparation was separated into nine fractions by reverse-phase HPLC on a $\mathrm{C} 18$ column (Figure 3(c)). Based on the results of Western blot analysis in Figure 3(d), fractions 1, 2, and 9 showed strong activity to downregulate LPS-induced iNOS expression. Interestingly, these active fractions showed comparable activity with the parent $90 \%$ QFY preparation, whereas the other fractions did not show much activity.
3.3. Ginsenosides in RS Were Identified as the Principal Active Compounds in $Q F Y$. To identify the active ingredients, seven herbal ingredients in QFY were processed into 90\% ethanol solutions and assayed for downregulating iNOS in microglia following the procedure described for the whole QFY formulation. As shown in Figure 4(a), 90\% ethanol extracts of DG (90\% DG) and RS (90\% RS) abolished LPS-induced iNOS expression but showed no effect against COX-2 induction. The $90 \%$ RS preparation appeared to be more potent than the $90 \%$ DG preparation, although less RS was used in the formula than DG. For this reason, the 90\% RS solution was selected for further bioactivity-guided fractionation. The compounds in the $90 \%$ RS preparation were separated by HPLC under the same conditions as described for the $90 \%$ QFY preparation (Figure 4(b)). The resulting fractions were assayed for the activity towards iNOS induction. As shown in Figure 4(c), fraction 9 (90\% RS-F9) effectively and selectively suppressed LPS-induced iNOS expression to a greater extent than the parent $90 \%$ RS solution.

To identify the specific ginsenosides, the $90 \%$ RS preparation and 90\% RS-F9 were separated on a C18 HPLC column and detected with a MALDI-TOF mass spectrometer. As shown in Figure 5(a), the 90\% RS-F9 fraction showed identical distribution of base peaks compared with the parent $90 \%$ RS solution within the elution time interval. As listed in Table 1, a total of 18 ginsenosides and one notoginsenoside were identified in the $90 \%$ RS-F9 fraction in a negative ion 


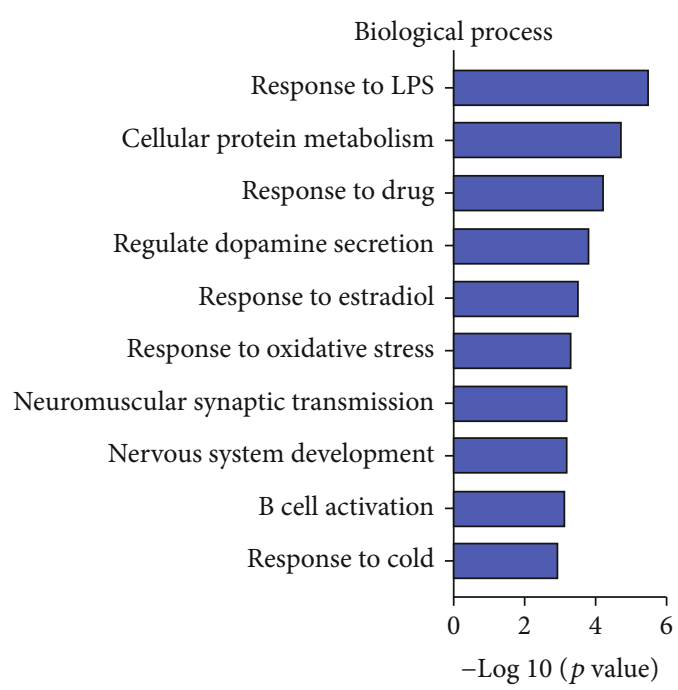

(a)

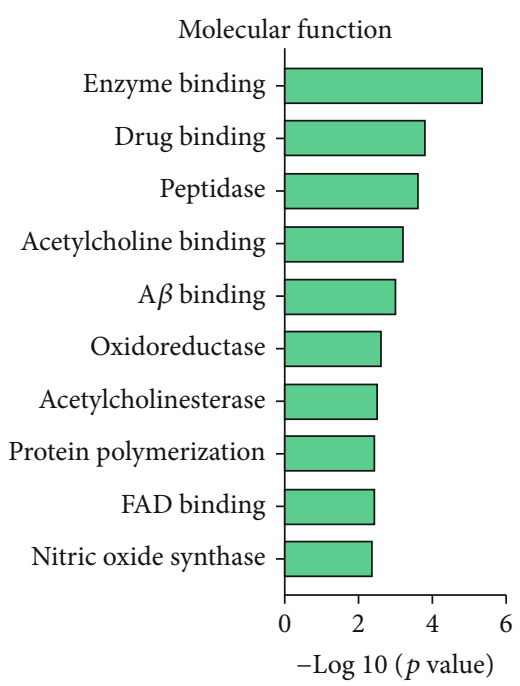

(b)

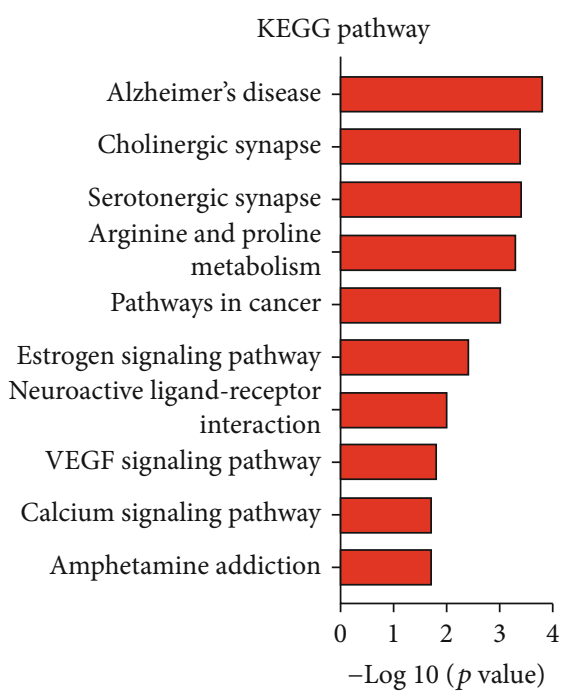

(c)

Figure 2: GO terms and KEGG pathway enrichment analysis of the protein targets. (a) The most significantly enriched GO terms were selected in terms of "biological process." (b) The most significantly enriched GO terms were selected in terms of "molecular function." (c) The most significantly enriched KEGG pathways were selected in terms of "KEGG pathways".

mode. In particular, peaks 19 and 20 were relatively prominent in the $90 \%$ RS-F9 fraction and detected as the stereoisomers of ginsenoside Rg3. 20(R)-Ginsenoside Rg3 and 20(S)ginsenoside were mainly detected as formate ions at $\mathrm{m} / \mathrm{z}$ 829.4965 and 829.4971 , respectively, while the deprotonated ions were also detected at $m / z 783.4906$ and 783.7922, respectively. To compare the migration pattern with pure compounds, commercial ginsenoside $\mathrm{Rg} 3$ was spiked in the 90\% RS-F9 fraction prior to LC-MS/MS analysis. As shown in Figure 5(b), ginsenoside $\mathrm{Rg} 3$ was verified by using the identical elution time and mass fragmentation pattern (data not shown). In addition, ginsenoside $\operatorname{Rg} 3$ was quantified from the acquired scan data using a target ion of 829.50. The calibration curve for ginsenoside $\operatorname{Rg} 3(y=62240.86 x-$ $\left.24991.72, R^{2}=0.9993\right)$ was optimized over the concentration range of $3.9-125 \mu \mathrm{g} / \mathrm{mL}$. The calculated amount of ginseno- side Rg3 in 90\% RS and 90\% RS-F9 was $2.11 \pm 0.02 \mathrm{mg} / \mathrm{g}$ and $2.05 \pm 0.08 \mathrm{mg} / \mathrm{g}$, respectively.

To examine the potency of different preparations to downregulate iNOS expression, ginsenoside Rg3 and the parent extracts (i.e., 90\% QFY and 90\% RS) were normalized based on quantification results in the assay of the in vitro effects. As shown in Figure 5(c), 90\% QFY and 90\% RS suppressed iNOS induction to an extent similar to $10 \mu \mathrm{M}$ and $20 \mu \mathrm{M}$ of ginsenoside $\mathrm{Rg} 3$, respectively.

\section{Discussion}

Microglial activation triggers neuroinflammation and neurodegeneration in the onset of AD [20]. Single-drug therapies such as nonsteroidal anti-inflammatory drugs (NSAIDs), TNF- $\alpha$ inhibitor, advanced glycation end product receptor 

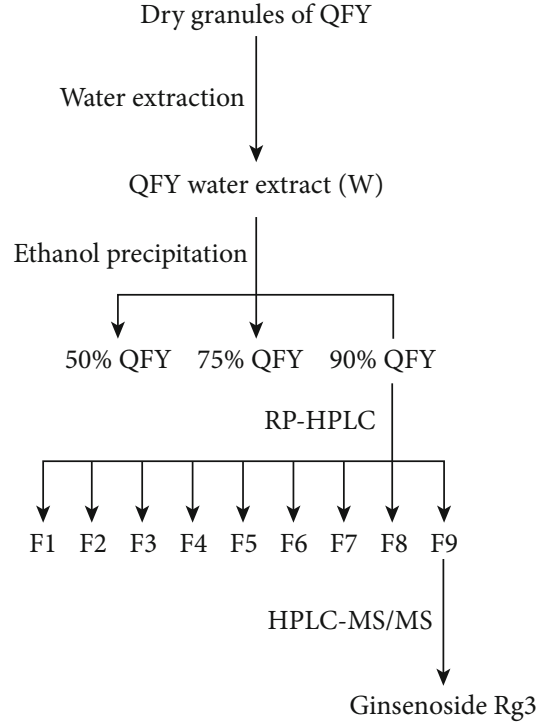

(a)

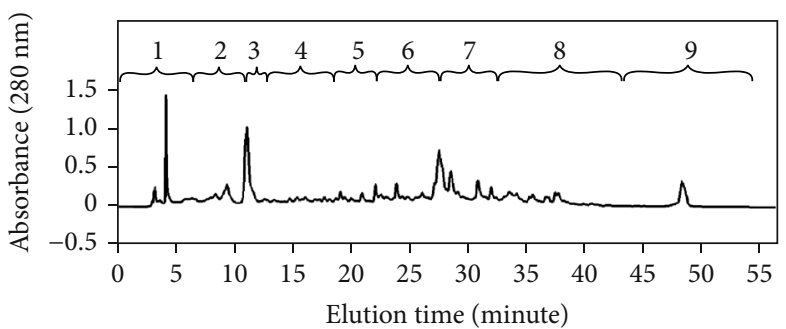

(c)
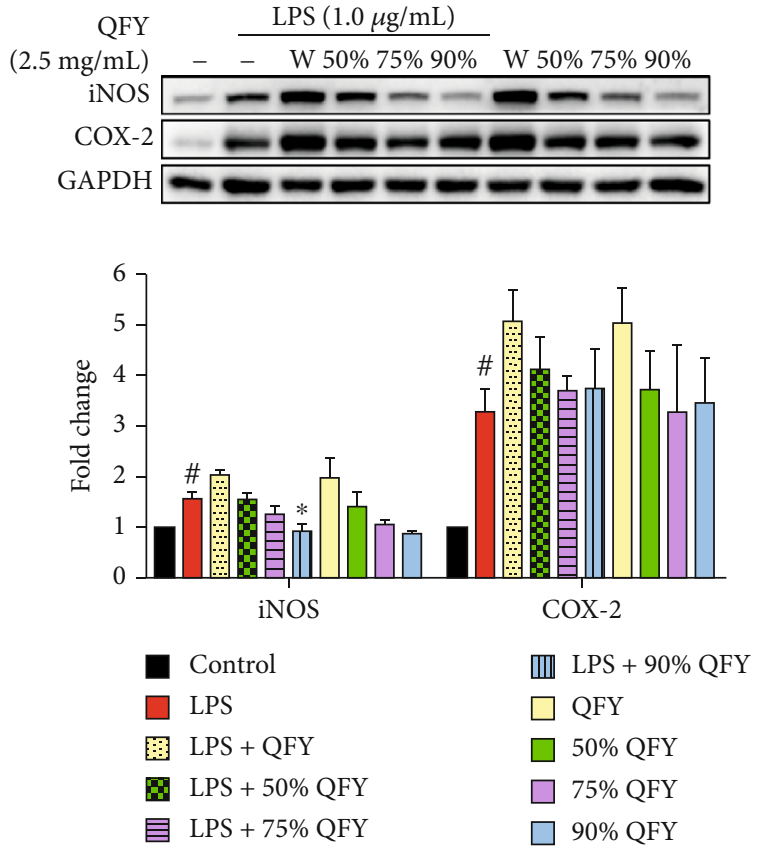

(b)
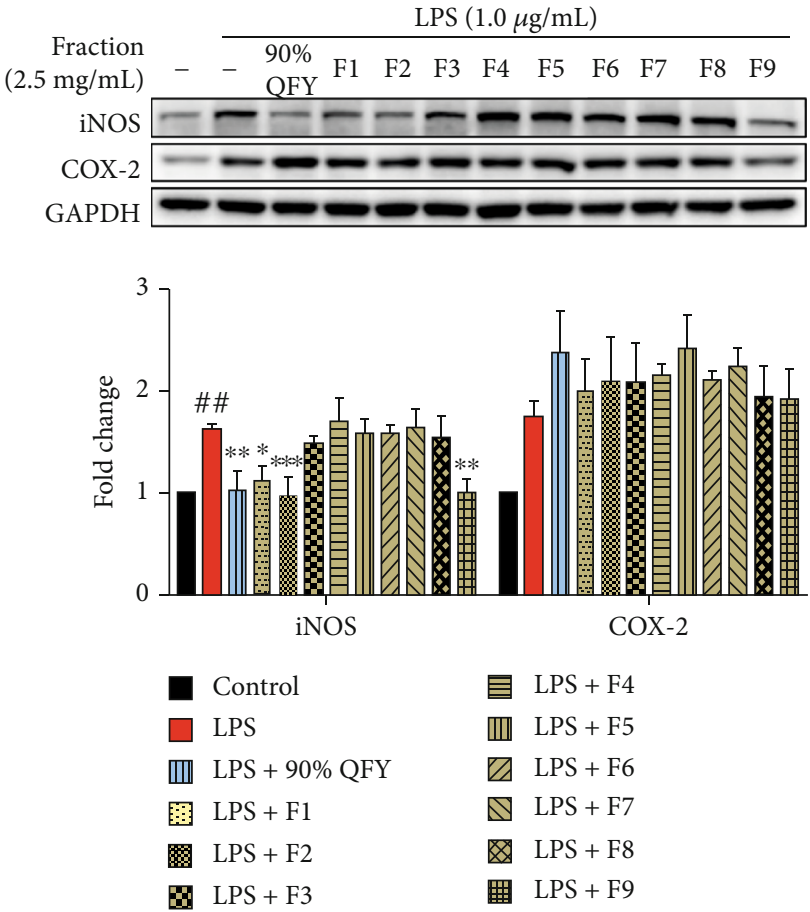

(d)

FIGURE 3: Bioactivity-guided fractionation of the herbal formulation QFY for potential active compounds. (a) Schematic illustration of the bioactivity-guided fractionation procedure. The fractions were prepared by sequential water extraction, ethanolic precipitation, and RP-HPLC separation on a C18 column. The fractions were sequentially analyzed by Western blot for the effects on LPS-induced expression of iNOS and COX-2 in BV-2 cells. (b) Bioassays of the QFY water extract and the fractions from ethanolic precipitation. BV-2 cells were treated with the indicated QFY extract with or without simultaneous stimulation with LPS for $24 \mathrm{~h}$. The expression of iNOS and COX-2 was detected by Western blot using specific antibodies. Representative blots were shown. The blots $(n=3)$ were quantified by a densitometric method using the ImageJ software. The results were expressed as mean \pm SD. ${ }^{\#} p<0.05$ (LPS vs. untreated control); ${ }^{*} p<0.05$ (treatment+LPS vs. LPS). (c) HPLC separation of the $90 \%$ QFY fraction. The compounds were separated into nine fractions by RP-HPLC on a C18 column. (d) Bioassays of the QFY-derived HPLC fractions. BV-2 cells were treated with the indicated concentration of different HPLC fractions or 90\% QFY together with LPS stimulation. The cellular proteins were analyzed by Western blot, and the blots were quantified as previously described. $\#$ \#\# 0.01 (LPS vs. untreated control); ${ }^{*} p<0.05,{ }^{* *} p<0.01$, and ${ }^{* * *} p<0.001$ (treatment+LPS vs. LPS). 

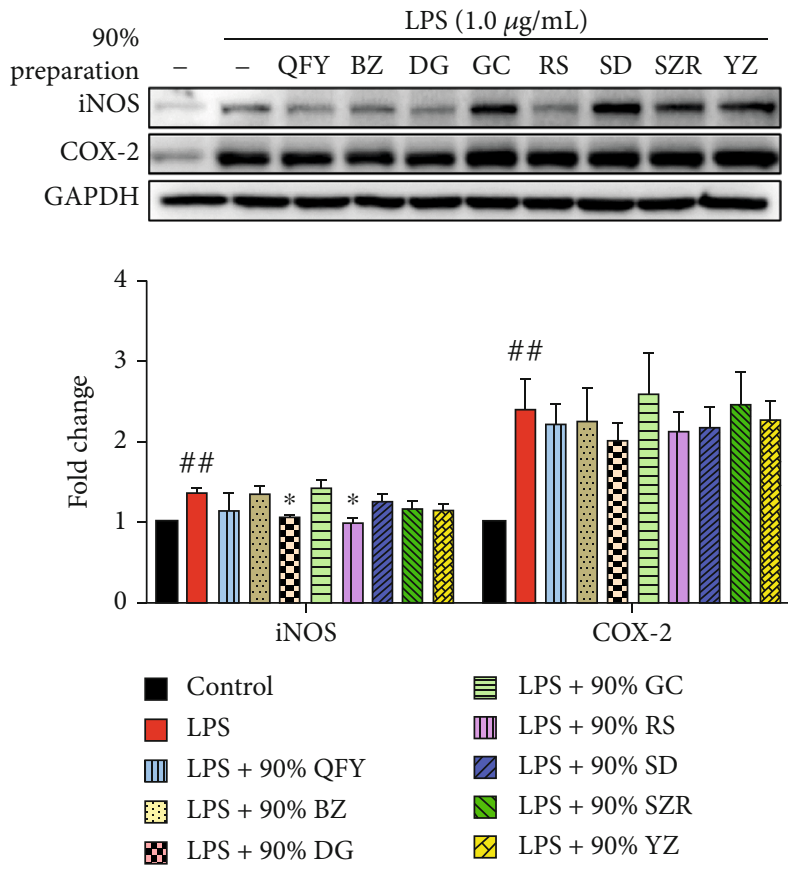

(a)

(b)
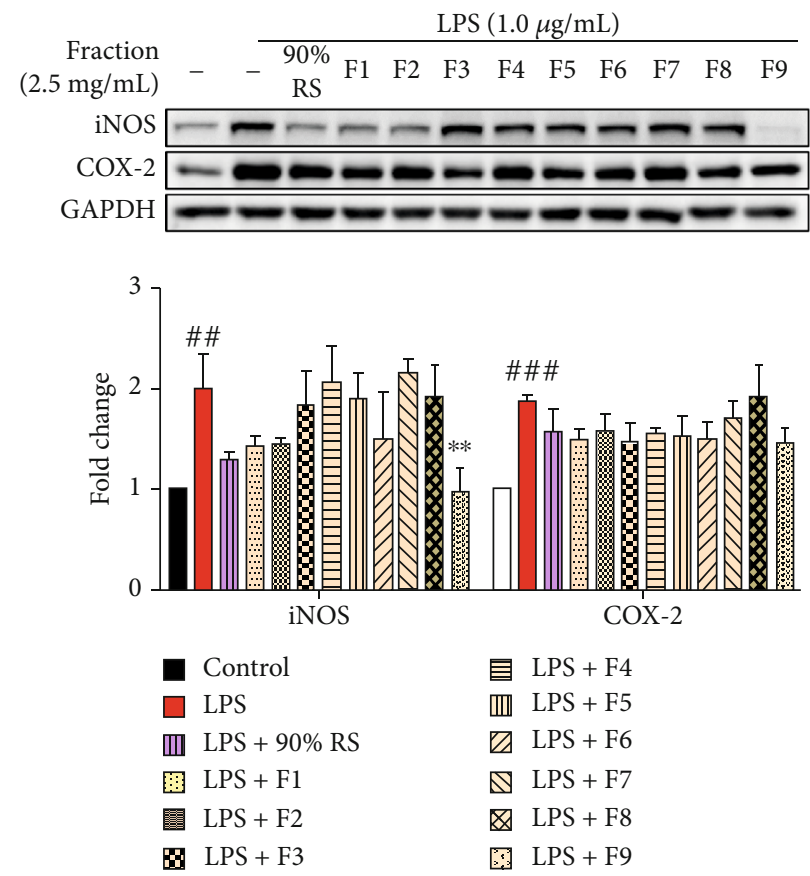

(c)

FIGURE 4: Identification of the active ingredients for the downregulation of iNOS expression. (a) Bioassays of the QFY extract and individual herbal extracts. QFY and individual herbal ingredients were extracted with water and precipitated with $90 \%$ ethanol. BV-2 cells were treated with $90 \%$ ethanol solutions of herbal extracts for $24 \mathrm{~h}$ with LPS stimulation. The expression of specific markers was detected by Western blot, and the blots were quantified as previously described. ${ }^{\# \#} p<0.01$ (LPS vs. untreated control); ${ }^{*} p<0.05$ (treatment+LPS vs. LPS). (b) HPLC separation of the $90 \%$ RS fraction. The $90 \%$ RS solution was separated into nine fractions by RP-HPLC on a C18 column using the same gradient and elution time as described for the 90\% QFY fraction. (c) Bioassays of the $90 \%$ RS-derived HPLC fractions. BV-2 cells were treated with the indicated concentration of HPLC fractions or $90 \%$ RS for $24 \mathrm{~h}$ with LPS stimulation. The expression of specific markers was detected by Western blot, and the blots were quantified as previously described. ${ }^{\#} p<0.01$, \#\#\# $p<0.001$ (LPS vs. untreated control); ${ }^{* *} p<0.01$ (treatment+LPS vs. LPS). 


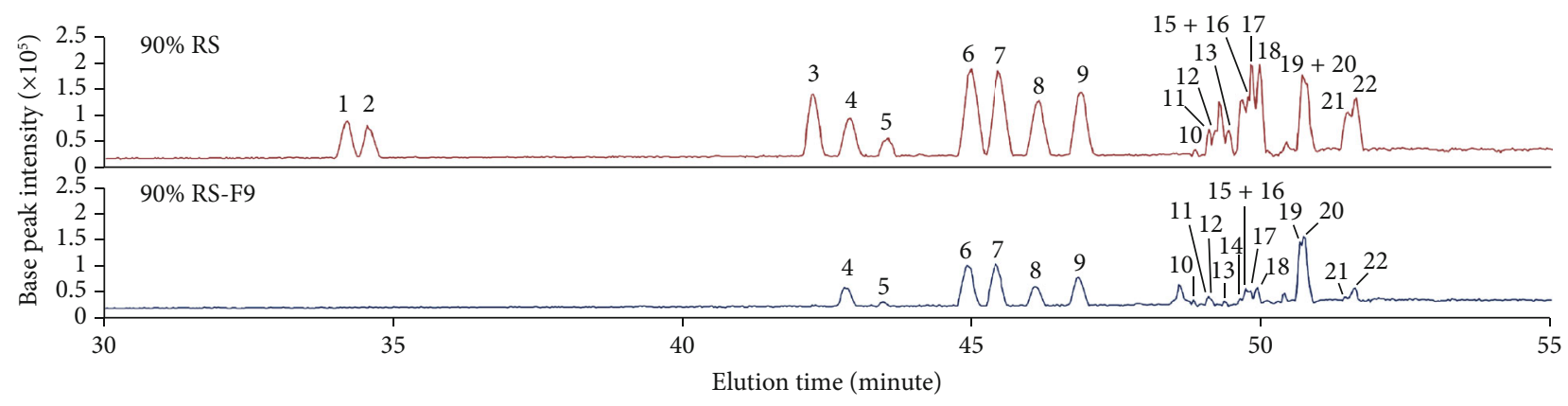

(a)

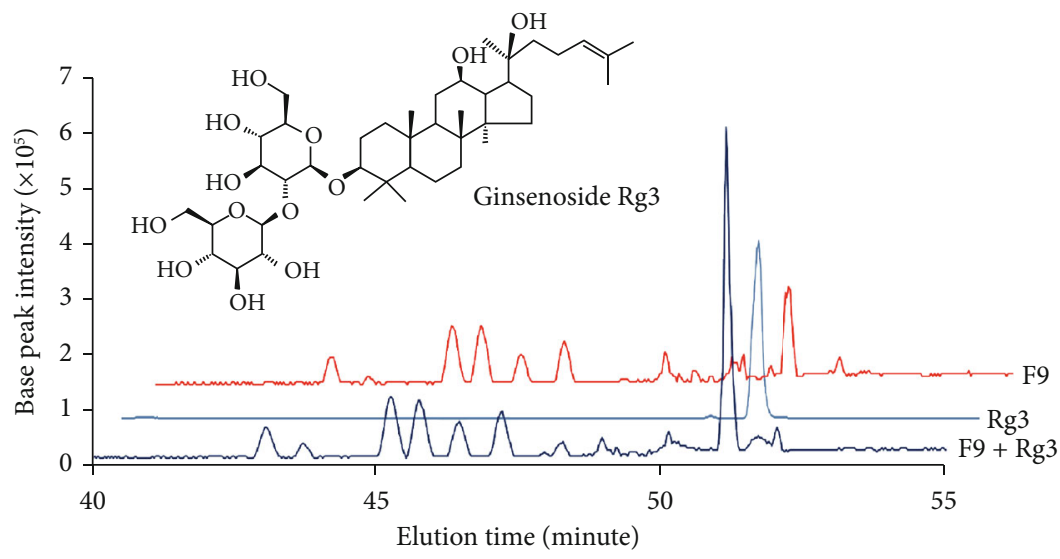

(b)

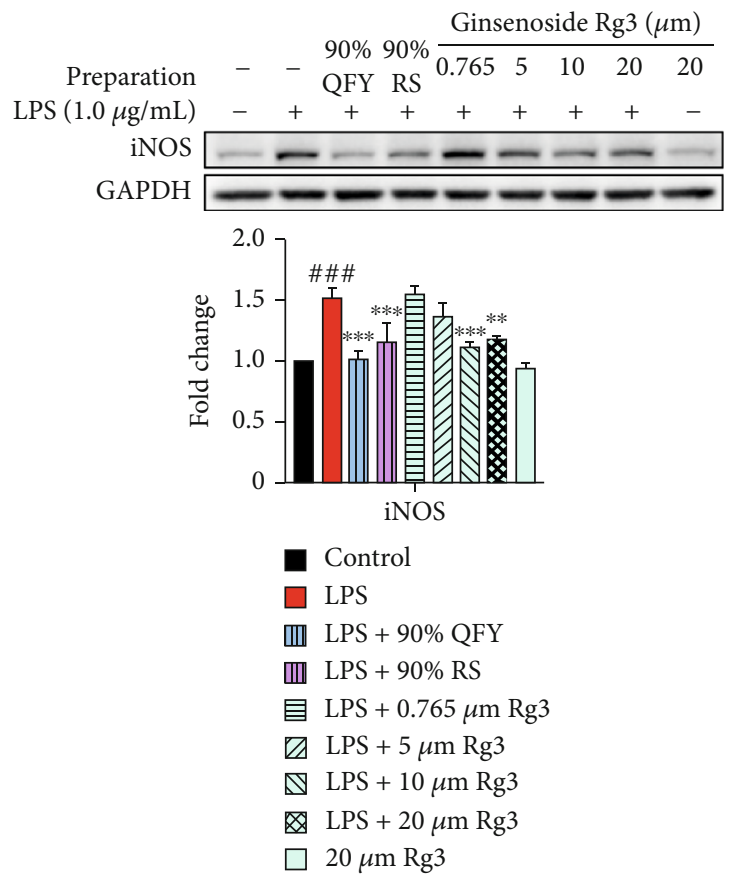

(c)

FIGURE 5: Chemical characterization of fraction 9 derived from $90 \%$ RS. (a) Chromatographic profiles of the parent $90 \%$ RS solution and the derived fraction 9. (b) Verification of ginsenoside $\operatorname{Rg} 3$ in the $90 \%$ RS-derived fraction 9. Fraction 9 and ginsenoside $\mathrm{Rg} 3$, alone or in combination, were analyzed by HPLC-MS/MS on a C18 column under the same conditions. (c) Bioassays of ginsenoside Rg3 for suppressing iNOS induction. BV-2 cells were treated with the indicated concentration of $90 \%$ QFY, $90 \%$ RS, and commercial ginsenoside Rg3 for $24 \mathrm{~h}$. The expression of iNOS was detected by Western blot, and the blots were quantified as previously described. \#\#\# $p<0.001$ (LPS vs. untreated control); ${ }^{* *} p<0.01,{ }^{* * *} p<0.001$ (treatment+LPS vs. LPS). 


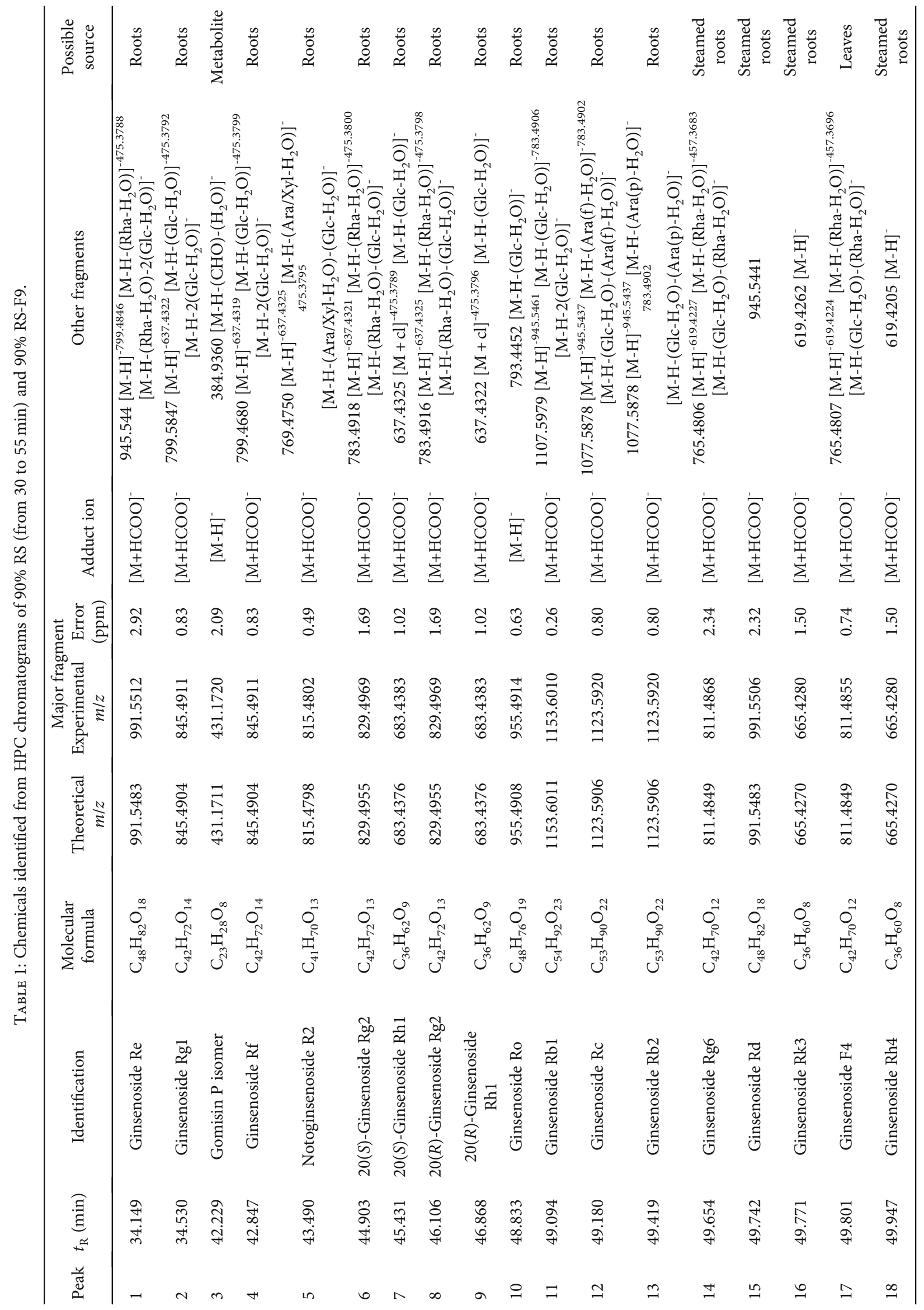




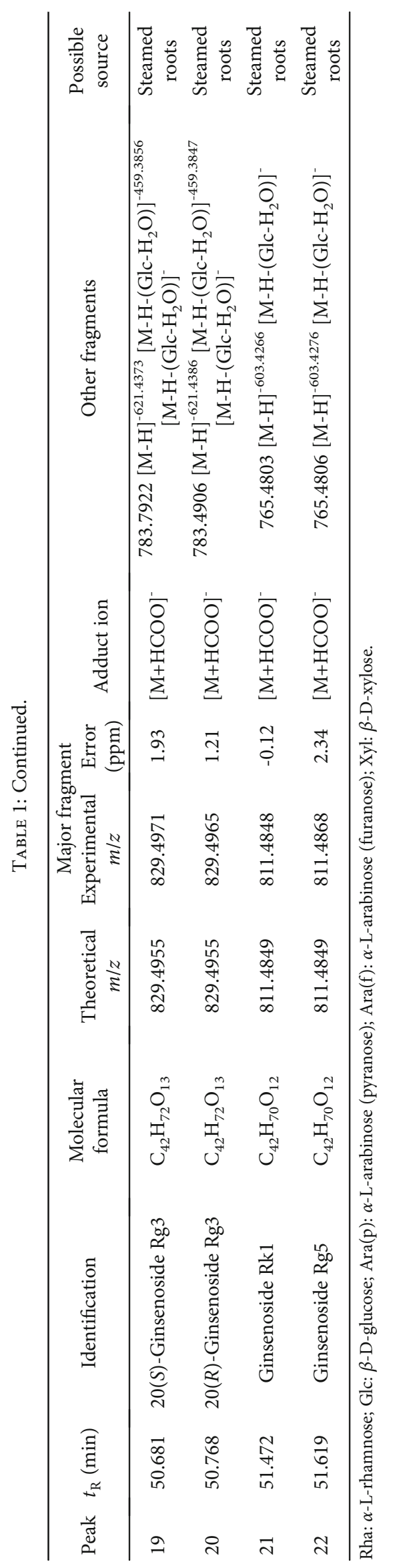


inhibitor, and PPAR $\gamma$ receptor agonist show varying efficacies in the treatment of $\mathrm{AD}$ [21]. Recent effort is directed to the discovery of multitarget drugs by a systems biology approach [22]. Many herbal formulas are well documented for the clinical treatment of dementia in traditional Chinese medicine, thereby representing a rich source for drug discovery [23]. The present study investigated the classical herbal formula QFY for the active compounds and protein targets and hence the molecular mechanisms for the management of $\mathrm{AD}$. We firstly employed a network pharmacology approach to predict the biological targets of QFY and the relevant signaling pathways. We further identified the active compounds from QFY for targeting the neuroinflammatory biomarker iNOS.

Network pharmacology encompasses systems biology, pharmacology, and computational algorithms to robustly study the complex drug-target relationships [24, 25]. Several herbal medicines including RS and YZ were previously studied for the molecular targets against AD by a network pharmacology approach [26]. In the present study, we employed a network pharmacology approach to study the action of the comprehensive anti-AD herbal formula QFY. Firstly, we shortlisted the active compounds with satisfactory bioavailability and BBB penetration based on the common pharmacokinetic or physiochemical parameters curated in databases. We secondly predicted 25 protein targets for these active compounds by target fishing. The C-T network in Figure 1(a) demonstrated that multiple compounds interacted with each protein, whereas the T-P network in Figure 1(b) also vividly depicted that the protein targets were involved in different pathways. Finally, the selected targets were subjected to GO and KEGG enrichment analysis. QFY may exert the pharmacological effect against $\mathrm{AD}$ by modulating $\mathrm{A} \beta$ aggregation, acetylcholinesterase activity, nitric oxide synthase activity, and cholinergic neurotransmission (Figure 2).

Neuroinflammation is well known to cause extensive damage to the central nervous system, leading to the disruption of synaptic function and the exacerbation of $\mathrm{A} \beta$ pathology [27]. Along this line, the present study focused on the antineuroinflammatory activity of QFY and selected two key proinflammatory biomarkers iNOS and COX-2 from the prediction of network pharmacology. It was previously demonstrated that both iNOS and COX-2 were overexpressed in the activated microglia and contributed to neurodegeneration [28]. To investigate the effect of QFY on the expression of iNOS and COX-2, we adopted a bioactivityguided fractionation approach to separate QFY into different fractions by water extraction, ethanolic precipitation, and HPLC fractionation. We prepared the QFY formulation from the dried, concentrated granules of different herbal ingredients, which facilitated the extraction process and produced a similar chemical profile to regular decoction [29]. We assayed all fractions for the activity to suppress LPSinduced expression of iNOS and COX-2 in microglial BV-2 cells. Based on Western blot analysis, the aqueous QFY extract potentiated the stimulatory effect of LPS on the expression of iNOS and COX-2. The stimulatory effect on microglia could be attributed to the water-soluble active immunopolysaccharide in the herbal ingredients [30]. On the other hand, alcoholic precipitation removed charged small molecules and polysaccharides from the aqueous herbal extracts that could stimulate the cells $[31,32]$. Indeed, the 90\% QFY preparation selectively suppressed LPSinduced iNOS expression in microglial BV-2 cells.

A bioactivity-guided fractionation approach is widely used to separate complex chemical mixture into different fractions, facilitating the identification of active compounds for specific biological entities [33-35]. In the present study, we firstly prepared a water extract and 50\%, 75\%, and $90 \%$ QFY ethanol extracts. Based on the bioassay results, the 90\% QFY preparation appeared to be the active fraction. Therefore, the $90 \%$ QFY preparation was subsequently separated into 9 fractions by HPLC (Figure 3(c)). Western blot analysis suggested that fractions 1,2 , and 9 effectively suppressed LPS-induced iNOS expression, although different chemicals might contribute to the activity (Figure 3(d)). For the identification of the active compounds, we screened seven herbal ingredients in the formulation QFY and identified that 90\% DG and 90\% RS preparations selectively suppressed iNOS induction in LPS-challenged BV-2 cells (Figure 4(a)). We selected the $90 \%$ RS preparation for further identification of the active compounds since RS-derived active compounds better met the requirements for an orally active drug in network pharmacology analysis. Thus, the $90 \%$ RS preparation was separated into 9 fractions by HPLC using the same gradient and fractionation as described for the 90\% QFY preparation. Western blot analysis suggested that fraction 9 derived from $90 \%$ RS best suppressed LPS-induced iNOS expression (Figures 4(b) and 4(c)). The 90\% RS and 90\% QFY preparations showed similar efficacy of inhibiting iNOS expression. In fact, previous studies demonstrated that RS exhibited excellent antineuroinflammatory and anti-A $\beta$ potency $[17,36]$. Based on the LC-MS/MS profile, fraction 9 might contain several antineuroinflammatory ginsenosides such as Rh1, Rb2, Rd, Rg3, and Rg5 [37-40]. In particular, ginsenoside Rg3 could downregulate iNOS against LPS stimulation in macrophages and promote the phenotypic switch of macrophages towards a proresolving M2 subtype [41, 42]. Indeed, LC-MS/MS analysis confirmed the presence of ginsenoside Rg3 in 90\% RS-derived fraction 9 since ginsenoside $\mathrm{Rg} 3$ spike-in comigrated with the endogenous ginsenoside Rg3 (Figure 5(b)). Furthermore, $10 \mu \mathrm{M}$ ginsenoside Rg3 appeared to achieve the same potency of $90 \%$ QFY and 90\% RS for suppressing iNOS induction, although ginsenoside $\mathrm{Rg} 3$ failed to exhibit similar activity at the concentration in $90 \%$ RS as deduced by the UPLC quantification (Figure 5(c)). Presumably, other compounds may act on iNOS induction in synergy with ginsenoside Rg3. As for the differential effects of QFY fractions on LPS-induced expression of iNOS and COX-2, we previously found that the concomitant activation of the antioxidant $\mathrm{Nrf} / \mathrm{HO}-1$ pathway was a potential mechanism to support the selective suppression of iNOS over COX-2 in macrophages [43]. As elevation of iNOS resulted in oxidative damage in neurons, activation of the Nrf2/HO-1 pathway could effectively inhibit inflammation and oxidative stress, which restored cellular function to the physiological state [44]. Previous studies reported that ginseng and ginsenoside $\mathrm{Rg} 3$ could activate the $\mathrm{Nrf} 2 / \mathrm{HO}-1$ pathway $[45,46]$. We speculate that ginsenoside $\mathrm{Rg} 3$ 
selectively suppressed iNOS induction in microglia due to the concomitant activation of the $\mathrm{Nrf} 2 / \mathrm{HO}-1$ pathway.

\section{Conclusions}

The present study demonstrated that the active compounds of the herbal formulation QFY could target several protein targets and signaling pathways in the pathogenesis of AD. Ginsenoside $\operatorname{Rg} 3$ was identified for potential activity to suppress LPS-induced iNOS expression in BV-2 microglial cells. Ultimately, the present study confirmed the possibility to identify the active compounds for targeting the most important antineuroinflammatory biomarkers against microglial activation and towards the development of anti-AD therapeutics.

\section{Data Availability}

The data supporting the findings of this study are available in the article and its supplementary materials.

\section{Conflicts of Interest}

The authors declare no conflict of interest.

\section{Authors' Contributions}

Fung Yin Ngo performed the in vitro experiments and drafted the manuscript. Weiwei Wang conducted the network pharmacology analysis. Qilei Chen conducted the mass spectrometry identification. Jin-Ming Gao and Hubiao Chen supervised the data analysis. Jia Zhao and Jianhui Rong designed the research. Jianhui Rong supervised the data analysis, secured the funds to support this study, and finalized the manuscript. All authors revised the manuscript and approved the submitted version. Fung Yin Ngo and Weiwei Wang contributed equally to this work.

\section{Acknowledgments}

This work was supported by General Research Fund (GRF) grants $(17120915,17146216,17100317$, and 17119619) from the Research Grants Council of Hong Kong, National Natural Science Foundation of China (nos. 81703726 and 21778046), Health and Medical Research Fund (15161731, 16171751, and 17181231), Science, Technology and Innovation Commission of Shenzhen Municipality (Basic Research Program, Free Exploration Project JCYJ20180306173835901), Research and Cultivation Plan of High-Level Hospital Construction (HKUSZH201902040), Midstream Research Programme for Universities (MRP) (053/18X), and Seed Funding for Basic Research Programme from the University of Hong Kong (201611159156).

\section{Supplementary Materials}

Table S1: Candidate compounds obtained from the TCMSP database and their pharmacokinetic parameters. Table S2: Candidate compounds obtained from the TCMID database and their Lipinski's rule of five parameters. (Supplementary Materials)

\section{References}

[1] M. W. Bondi, E. C. Edmonds, and D. P. Salmon, "Alzheimer's disease: past, present, and future," Journal of the International Neuropsychological Society, vol. 23, no. 9-10, pp. 818-831, 2017.

[2] M. Goedert, "Alzheimer's and Parkinson's diseases: the prion concept in relation to assembled $\mathrm{A} \beta$, tau, and $\alpha$-synuclein," Science, vol. 349, no. 6248, p. 1255555, 2015.

[3] C. G. Parsons, W. Danysz, A. Dekundy, and I. Pulte, "Memantine and cholinesterase inhibitors: complementary mechanisms in the treatment of Alzheimer's disease," Neurotoxicity Research, vol. 24, no. 3, pp. 358-369, 2013.

[4] K. G. Yiannopoulou and S. G. Papageorgiou, "Current and future treatments for Alzheimer's disease," Therapeutic Advances in Neurological Disorders, vol. 6, no. 1, pp. 19-33, 2012.

[5] M. T. Heneka, M. J. Carson, J. E. Khoury et al., "Neuroinflammation in Alzheimer's disease," The Lancet Neurology, vol. 14, no. 4, pp. 388-405, 2015.

[6] S. Hickman, S. Izzy, P. Sen, L. Morsett, and J. El Khoury, "Microglia in neurodegeneration," Nature Neuroscience, vol. 21, no. 10, pp. 1359-1369, 2018.

[7] M. L. Block and J. S. Hong, "Microglia and inflammationmediated neurodegeneration: multiple triggers with a common mechanism," Progress in Neurobiology, vol. 76, no. 2, pp. 77-98, 2005.

[8] T. Wei, C. Chen, J. Hou, W. Xin, and A. Mori, "Nitric oxide induces oxidative stress and apoptosis in neuronal cells," Biochimica et Biophysica Acta (BBA) - Molecular Cell Research., vol. 1498, no. 1, pp. 72-79, 2000.

[9] W.-Y. Fu, X. Wang, and N. Y. Ip, "Targeting neuroinflammation as a therapeutic strategy for Alzheimer's disease: mechanisms, drug candidates, and new opportunities," ACS chemical neuroscience., vol. 10, no. 2, pp. 872-879, 2019.

[10] L. Lin, Z. Lan, and Y. Cui-cui, "Multi-target strategy and experimental studies of traditional Chinese medicine for Alzheimer's disease therapy," Current Topics in Medicinal Chemistry, vol. 16, no. 5, pp. 537-548, 2016.

[11] W.-Y. Ong, Y.-J. Wu, T. Farooqui, and A. A. Farooqui, "Qi Fu Yin-a Ming dynasty prescription for the treatment of dementia," Molecular Neurobiology, vol. 55, no. 9, pp. 7389-7400, 2018.

[12] S. Y. Wang, J. P. Liu, W. W. Ji et al., "Qifu-Yin attenuates AGEs-induced Alzheimer-like pathophysiological changes through the RAGE/NF- $\kappa$ B pathway," Chinese journal of natural medicines., vol. 12, no. 12, pp. 920-928, 2014.

[13] G. H. Xing, C. R. Lin, N. Hu, Y. C. Niu, and Y. R. Sun, "Effect of Qifuyin on ability of learning and memory and expression of somatostatin in hippocampus on model rats of Alzheimer's disease induced by $\beta$-amyloid 1-42," Chinese Journal of Information on Traditional Chinese Medicine, vol. 17, pp. 34-36, 2010.

[14] J. P. Liu, Q. Q. Wang, Z. M. Hu, S. Y. Wang, and S. P. Ma, "Effect the AGEs/AGE/NF- $\kappa$ B pathway in Alzheimer's disease model rats of Qifu Yin," Pharmacology and Clinics of Chinese Materia Medica, vol. 31, pp. 9-11, 2015.

[15] M.-N. Li, X. Dong, W. Gao et al., "Global identification and quantitative analysis of chemical constituents in traditional Chinese medicinal formula Qi-Fu-Yin by ultra-high performance liquid chromatography coupled with mass spectrometry," 
Journal of Pharmaceutical and Biomedical Analysis, vol. 114, pp. 376-389, 2015.

[16] H.-M. An, D.-R. Huang, H. Yang et al., "Comprehensive chemical profiling of Jia-Wei-Qi-Fu-Yin and its network pharmacology-based analysis on Alzheimer's disease," Journal of Pharmaceutical and Biomedical Analysis, vol. 189, p. 113467, 2020.

[17] J. H. Kim, Y.-S. Yi, M.-Y. Kim, and J. Y. Cho, "Role of ginsenosides, the main active components of Panax ginseng, in inflammatory responses and diseases," Journal of Ginseng Research, vol. 41, no. 4, pp. 435-443, 2017.

[18] M. H. Cheong, S. R. Lee, H. S. Yoo et al., "Anti-inflammatory effects of Polygala tenuifolia root through inhibition of NF$\kappa \mathrm{B}$ activation in lipopolysaccharide-induced BV2 microglial cells," Journal of Ethnopharmacology, vol. 137, no. 3, pp. 1402-1408, 2011.

[19] J. Y. Yu, J. Y. Ha, K. M. Kim, Y. S. Jung, J. C. Jung, and S. Oh, "Anti-inflammatory activities of licorice extract and its active compounds, glycyrrhizic acid, liquiritin and liquiritigenin, in BV2 cells and mice liver," Molecules, vol. 20, no. 7, pp. 13041-13054, 2015.

[20] M. L. Block, L. Zecca, and J.-S. Hong, "Microglia-mediated neurotoxicity: uncovering the molecular mechanisms," Nature Reviews Neuroscience, vol. 8, no. 1, pp. 57-69, 2007.

[21] Y. Dong, X. Li, J. Cheng, and L. Hou, "Drug development for Alzheimer's disease: microglia induced neuroinflammation as a target?," International Journal of Molecular Sciences, vol. 20, no. 3, p. 558, 2019.

[22] A. L. Hopkins, "Network pharmacology: the next paradigm in drug discovery," Nature Chemical Biology, vol. 4, no. 11, pp. 682-690, 2008.

[23] Z.-K. Sun, H.-Q. Yang, and S.-D. Chen, "Traditional Chinese medicine: a promising candidate for the treatment of Alzheimer's disease," Translational Neurodegeneration, vol. 2, no. 1, p. 6, 2013.

[24] X. Liang, H. Li, and S. Li, "A novel network pharmacology approach to analyse traditional herbal formulae: the LiuWei-Di-Huang pill as a case study," Molecular BioSystems, vol. 10, no. 5, pp. 1014-1022, 2014.

[25] S. Li and B. Zhang, "Traditional Chinese medicine network pharmacology: theory, methodology and application," Chinese Journal of Natural Medicines, vol. 11, no. 2, pp. 110-120, 2013.

[26] J. Fang, L. Wang, T. Wu et al., "Network pharmacology-based study on the mechanism of action for herbal medicines in Alzheimer treatment," Journal of Ethnopharmacology, vol. 196, pp. 281-292, 2017.

[27] F. L. Heppner, R. M. Ransohoff, and B. Becher, "Immune attack: the role of inflammation in Alzheimer disease," Nature Reviews Neuroscience, vol. 16, no. 6, pp. 358-372, 2015.

[28] M. N. Catorce and G. Gevorkian, "LPS-induced murine neuroinflammation model: main features and suitability for preclinical assessment of nutraceuticals," Current Neuropharmacology, vol. 14, no. 2, pp. 155-164, 2016.

[29] L. Chen, Q. Wang, and J. Liu, "Simultaneous analysis of nine active components in Gegen Qinlian preparations by highperformance liquid chromatography with diode array detection," Journal of Separation Science, vol. 29, no. 14, pp. 22032210, 2006.

[30] Y. Ji, R. Wang, Y. Peng, C. Peng, and X. Li, "Purification, preliminary characterization, and immunological activity of polysaccharides from crude drugs of Sijunzi formula," Evidence-
Based Complementary and Alternative Medicine, vol. 2017, Article ID 2170258, 8 pages, 2017.

[31] G. Y. Koh, G. Chou, and Z. Liu, "Purification of a water extract of Chinese sweet tea plant (Rubus suavissimus S. Lee) by alcohol precipitation," Journal of Agricultural and Food Chemistry, vol. 57, no. 11, pp. 5000-5006, 2009.

[32] X. Yang, Y. Zhao, Y. Yang, and Y. Ruan, "Isolation and characterization of immunostimulatory polysaccharide from an herb tea, Gynostemma pentaphyllum Makino," Journal of Agricultural and Food Chemistry, vol. 56, no. 16, pp. 69056909, 2008.

[33] M. G. Weller, "A unifying review of bioassay-guided fractionation, effect-directed analysis and related techniques," Sensors, vol. 12, no. 7, pp. 9181-9209, 2012.

[34] H. Qi, S. O. Siu, Y. Chen et al., "Senkyunolides reduce hydrogen peroxide-induced oxidative damage in human liver HepG2 cells via induction of heme oxygenase-1," ChemicoBiological Interactions, vol. 183, no. 3, pp. 380-389, 2010.

[35] C. Yang, J. Zhao, Y. Cheng, X. Li, and J. Rong, "Bioactivityguided fractionation identifies amygdalin as a potent neurotrophic agent from herbal medicine Semen Persicae extract," BioMed Research International, vol. 2014, Article ID 306857, 10 pages, 2014.

[36] W.-Y. Ong, T. Farooqui, H.-L. Koh, A. A. Farooqui, and E.A. Ling, "Protective effects of ginseng on neurological disorders," Frontiers in Aging Neuroscience, vol. 7, no. 129, 2015.

[37] J. S. Jung, J. A. Shin, E. M. Park et al., "Anti-inflammatory mechanism of ginsenoside Rh1 in lipopolysaccharidestimulated microglia: critical role of the protein kinase A pathway and hemeoxygenase-1 expression," Journal of Neurochemistry, vol. 115, no. 6, pp. 1668-1680, 2010.

[38] C. F. Wu, X. L. Bi, J. Y. Yang et al., "Differential effects of ginsenosides on NO and TNF- $\alpha$ production by LPS-activated N9 microglia," International Immunopharmacology, vol. 7, no. 3, pp. 313-320, 2007.

[39] S. S. Joo, Y. M. Yoo, B. W. Ahn et al., "Prevention of inflammation-mediated neurotoxicity by $\mathrm{Rg} 3$ and its role in microglial activation," Biological \& Pharmaceutical Bulletin, vol. 31, no. 7, pp. 1392-1396, 2008.

[40] S. Chu, J. Gu, L. Feng et al., "Ginsenoside Rg5 improves cognitive dysfunction and beta-amyloid deposition in STZ-induced memory impaired rats via attenuating neuroinflammatory responses," International Immunopharmacology, vol. 19, no. 2, pp. 317-326, 2014.

[41] S. Kang, S.-J. Park, A.-Y. Lee, J. Huang, H.-Y. Chung, and D.S. Im, "Ginsenoside Rg3 promotes inflammation resolution through M2 macrophage polarization," Journal of Ginseng Research, vol. 42, no. 1, pp. 68-74, 2018.

[42] S.-J. Yoon, J.-Y. Park, S. Choi et al., “Ginsenoside Rg3 regulates S-nitrosylation of the NLRP3 inflammasome via suppression of iNOS," Biochemical and Biophysical Research Communications, vol. 463, no. 4, pp. 1184-1189, 2015.

[43] Y. Cheng, C. Yang, D. Luo, and X. Li, "N-Propargyl caffeamide skews macrophages towards a resolving M2-like phenotype against myocardial ischemic injury via activating $\mathrm{Nrf} 2 / \mathrm{HO}-1$ pathway and inhibiting NF- $\kappa \mathrm{B}$ pathway," Cellular Physiology and Biochemistry, vol. 47, no. 6, pp. 2544-2557, 2018.

[44] J.-F. Luo, X.-Y. Shen, C. K. Lio et al., "Activation of Nrf2/HO-1 pathway by nardochinoid $\mathrm{C}$ inhibits inflammation and oxidative stress in lipopolysaccharide-stimulated macrophages," Frontiers in Pharmacology, vol. 9, p. 911, 2018. 
[45] G. Carota, M. Raffaele, V. Sorrenti, L. Salerno, V. Pittalà, and S. Intagliata, "Ginseng and heme oxygenase-1: the link between an old herb and a new protective system," Fitoterapia, vol. 139, p. 104370, 2019.

[46] C. K. Lee, K.-K. Park, A.-S. Chung, and W.-Y. Chung, "Ginsenoside $\mathrm{Rg} 3$ enhances the chemosensitivity of tumors to cisplatin by reducing the basal level of nuclear factor erythroid 2-related factor 2-mediated heme oxygenase-1/NAD(P)H quinone oxidoreductase- 1 and prevents normal tissue damage by scavenging cisplatin-induced intracellular reactive oxygen species," Food and Chemical Toxicology, vol. 50, no. 7, pp. 25652574, 2012. 